\title{
Directive mmWave radio channel modelling in a ship hull
}

\author{
BRECHT DE BEELDE ${ }^{1}$, ANDRÉS ALMARCHA LOPÉZ ${ }^{1,2}$, DAVID PLETS ${ }^{1}$, MARWAN YUSUF ${ }^{1}$, \\ EMMERIC TANGHE ${ }^{1}$ AND WOUT JOSEPH ${ }^{1}$
}

\begin{abstract}
Wireless connectivity has been realized for multiple environments and different frequency bands. However, little research exists about mmWave communication in industrial environments. This paper presents the $60 \mathrm{GHz}$ double-directional radio channel for mmWave communication in a ship hull for Line-of-Sight (LOS) and non Line-of-Sight (NLOS) conditions. We performed channel measurements using the Terragraph channel sounder at different locations in the ship hull and fitted LOS path loss to a one-slope path loss model. Path loss and root-mean-square (RMS) delay spread of the LOS path is compared to the reflected path with lowest path loss. NLOS communication via this first-order reflected path is modelled by calculating the path distance and determining the reflection loss. The reflection losses have a considerable contribution to the signal attenuation of the reflected path. The channel models are implemented in an indoor coverage prediction tool, which was extended with a ray launching algorithm and validated by comparison with an analytical electromagnetic solver. The results show that the mmWave radio channel allows high-throughput communication within a ship hull compartment, even when no LOS path between the transmitter and receiver is present.
\end{abstract}

Keywords: mmWave, $60 \mathrm{GHz}$, propagation, channel modelling, ship hull, coverage prediction, ray launching

\section{INTRODUCTION}

Wireless communication is omnipresent in our daily lives, from wearables in personal area networks to laptops in wireless local area networks and phones in broadband cellular networks. In order to optimize wireless communication, radio channel models for various environments and frequency bands have been developed, ranging from office environments [1-6] to outdoor [7-11] and industrial [1215] environments. As the sub- $6 \mathrm{GHz}$ industrial, scientific and medical (ISM) bands are getting crowded and future applications require higher data rates, development on mmWave channel models and radios is ongoing $[3,4,16-$ 22]. Most existing research investigates communication at mmWave frequencies in office or residential environments. However, wireless communication at mmWave frequencies also has a huge potential for industrial environments, such as low latency communication for valve control of machinery, video monitoring in remote areas or a virtual reality operator providing assistance to machine operators.

The maritime industry is converging to a smart transportation ecosystem consisting of interconnected devices resulting in partially automated or fully autonomous ships [23], with fewer crew members and increased on-board

\footnotetext{
${ }^{1}$ Ghent University/IMEC, Ghent, Belgium

${ }^{2}$ Barcelona Supercomputing Center, Barcelona, Spain
}

efficiency [24]. This paper considers the radio channel in a ship hull, a dangerous area where crew members are disconnected from the officers in the bridge and work in difficult circumstances. Wireless communication enhances the efficiency on board of the vessel by enabling automatic monitoring of equipment and therefore limiting the number of times crew members need to be in the ship hull [24]. It also increases safety by enabling video stream monitoring so an injured worker will be noticed faster and it can also replace existing wired communication links. The cables used for telecommunication and data transfer add to the deployment cost, noting that maritime cables should be corrosion resistant, fire and flame retardant and certified [25]. By enabling wireless communication systems, the number of cables deployed in the vessel can be reduced, which not only decreases the weight of the vessel, but also decreases the cost and simplifies maintenance, as finding and replacing a broken cable is not straightforward.

Most research on maritime wireless communication addresses off-ship communication [26-31]. Sub-6 GHz radio channel measurements on board of a vessel were carried out in [32-34], reporting a path loss exponent value of less than 2 for propagation within a metal compartment, which is in line with several other industrial environments [14]. Higher layer experiments of IEEE 802.15.4 based sensor networks on board of a vessel show a very good network reliability [35]. Modelling of $60 \mathrm{GHz}$ indoor radio channels is already presented in [4, 36-39]. 
In previous work we presented path loss models at sub$6 \mathrm{GHz}$ frequencies $868 \mathrm{MHz}, 2.4 \mathrm{GHz}$ and $5.25 \mathrm{GHz}$ [34], and a Line-of-Sight (LOS) path model at $60 \mathrm{GHz}[34,40]$. The $60 \mathrm{GHz}$ LOS path loss model from [40] is extended with a beamwidth-dependent PL model in [34]. The novelty of this paper is the analysis of the non Line-of-Sight (NLOS) channel model, investigation of delay spreads, and the channel model implementation in a ray launching network planning tool. In our network planning tool, the transmitted signal is represented by a set of planar wavefronts where the attenuation is based on the channel model and the angular information is based on the room geometry and locations of the transmitter and receiver. Ray launching algorithms for determining channel characteristics and predicting network performance at mmWave frequencies are investigated in multiple papers [41-45]. Most ray launching implementations consider transmission, specular reflection and diffraction. Even though diffuse scattering accounts for up to $26 \%$ of the total received power [46], it is not considered in most ray launching implementations [41].

In Section II we first present the equipment and setups of the measurement campaign. We then provide the channel modelling and link budget methodology, followed by a presentation of the channel model implementation in a network planning tool for predicting network performance and conclude the section with a validation of the network planning tool. Section III starts with a presentation of the LOS and NLOS channel modelling results and a link budget analysis based on the channel modelling results. We then provide the validation of the network planning tool and present network prediction simulation results for the ship hull environment. We conclude this paper in Section IV.

\section{METHODOLOGY}

We design a stochastic radio channel model for mmWave propagation in a ship hull, based on measurement campaigns we performed in an ocean-sailing vessel, and implement the channel model in a network planning tool.

\section{A) Channel sounder}

We use the Terragraph channel sounder which consists of two nodes, each containing a grid of $36 \times 8$ vertically polarized antennas with phase shifters and a Qualcomm QCA6438 transceiver chipset. The channel sounder is used by other institutions for outdoor $60 \mathrm{GHz}$ channel modelling [23, 47-49]. The spacing of the antenna elements is $0.55 \lambda$. The antenna grid results in a $-3 \mathrm{~dB}$ beam width of 2.8 degrees, which we can steer in the azimuth plane from -45 to +45 degrees with respect to the node's boresight, in steps of 1.4 degrees. The channel sounder successively transmits IEEE 802.11ad frames, at channel 2 with center frequency $60.48 \mathrm{GHz}$ and bandwidth $2.16 \mathrm{GHz}$, for each transmitter (TX) and receiver (RX) azimuthal beam configuration and performs a power measurement of the received

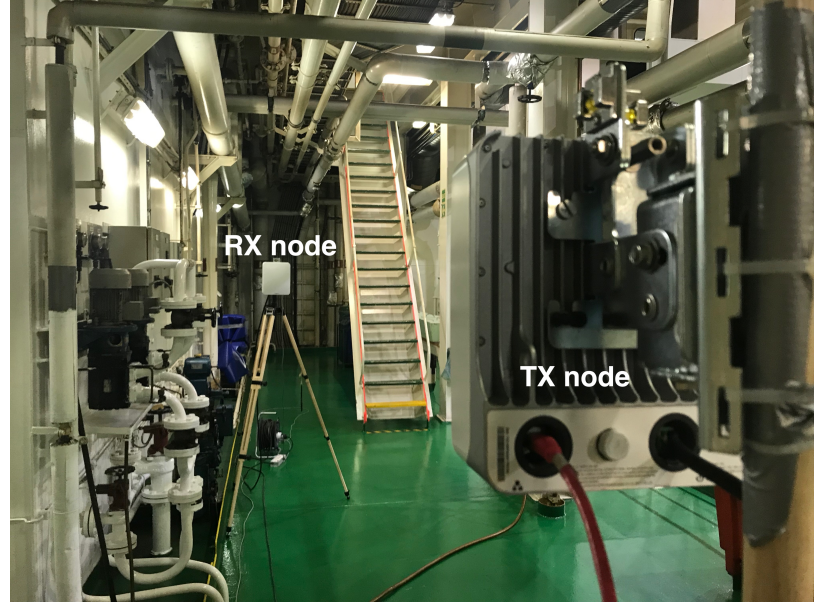

Fig. 1.: Terragraph channel sounder consisting of two nodes at distance $3.75 \mathrm{~m}$ along measurement track 1 in the engine room, synchronized via UTP and each having an antenna array of 288 antenna elements which allows beam steering in the azimuth plane.

signal. This results in a path loss angular profile (PLAP) that shows measured path loss as a function of both TX and $\mathrm{RX}$ azimuth angles, referred to as angle of departure (AoD) and angle of arrival (AoA) in the remainder of this paper. Sidelobes are present in the measured PLAP as the interelement spacing is larger than $\lambda / 2$ and there is only a phase adjustment for the antenna elements without gain tapering. The channel sounder is calibrated using a National Instruments mmWave transceiver. During the calibration, the chipset's reported received signal strength indicator (RSSI) is mapped to received power as a function of chip temperature, RX gain settings and antenna configuration. Similarly, TX gain settings are mapped to equivalent isotropically radiated power (EIRP). During the measurements, the RSSI measurement data and transceiver configuration (antennas, TX and RX gains, chip temperature) are stored on a laptop for offline processing, during which the measured signal power is interpolated with calibration data resulting in corrected received power. Validation measurements of the channel sounder in a laboratory setting show an uncertainty of less than $1 \mathrm{~dB}$ in measured PL.

The channel sounder performs a 256-tap complex channel impulse response (CIR) estimation based on the training sequence of the IEEE 802.11ad preamble, using sampling rate of $3.52 \mathrm{GHz}$. The CIR estimation results in the root-mean-squared (RMS) delay spread with a time resolution of $0.28 \mathrm{~ns}$. Both nodes are synchronized via an unshielded twisted pair (UTP) cable and the transmit power of $5 \mathrm{dBm}$ results in an equivalent isotropically radiated power (EIRP) of $45 \mathrm{dBm}$. The dynamic range of the sounder is $50 \mathrm{~dB}$. Figure 1 shows a picture of the channel sounder, with a distance of $3.75 \mathrm{~m}$ between the TX and RX nodes. 


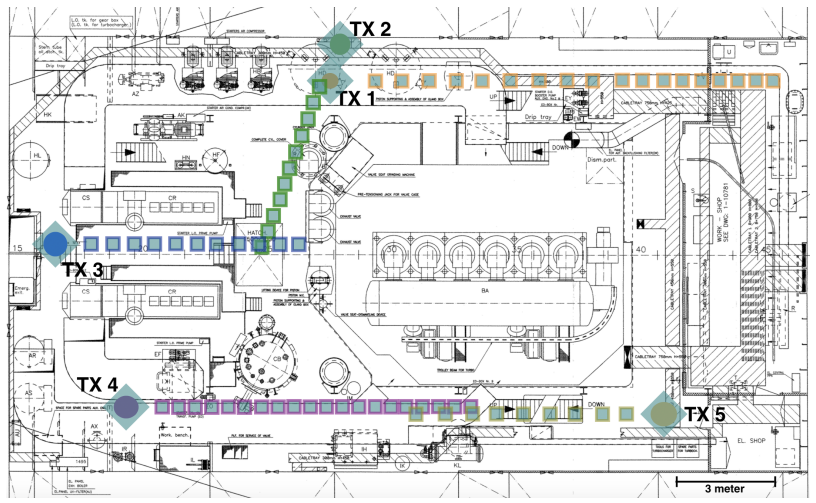

Fig. 2.: Floorplan of the vessel's engine room with transmitter (TX, diamond symbols) and receiver (RX, rectangular symbols) locations for the Line-of-Sight measurements.

\section{B) Measurement setup}

We performed LOS and NLOS measurements in the engine room and steering gear room of a $205 \mathrm{~m}$ long bulk carrier vessel with a gross tonnage of 33005. Measurements are performed along multiple tracks with increasing distance between the two nodes. For every track, the location of the TX node is fixed, while the RX node is moved away from the TX node.

During all measurements, the ship was moored in the harbour and no people were present in the engine and steering gear room. The vessel's main engine was not active, but an auxiliary engine used for electricity production was running. Even though this auxiliary engine was causing slight vibrations, the environment is considered static as there were no objects moving significantly and the vibrations due to the engine were much faster than the sweep time of the channel sounder.

\section{1) Line-of-Sight}

The locations of the LOS measurements in the engine room are shown in Fig. 2, in which the diamond markers indicate TX node locations and rectangular markers indicate RX node locations. The spacing of the nodes is chosen so that we have at least one PLAP measurement for every $0.25 \mathrm{~m}$ and at least two PLAP measurements on different tracks every $0.5 \mathrm{~m}$. When combining all measurements, we obtain PL as a function of distance for distances ranging from $1.5 \mathrm{~m}$ to $14.25 \mathrm{~m}$ with a spacing of $0.25 \mathrm{~m}$.

\section{2) Non Line-of-Sight}

The LOS measurements provide NLOS information as every measurement consists of an azimuth scan, but we also performed NLOS measurements where the LOS path is obstructed and where we rotated the nodes in order to capture the most significant reflected paths. In the engine room, the receiving node was placed behind the corner (shown in Fig. 8a) whereas in the steering gear room the large rotary vane connected to the vessel's rudder blocked the LOS path (shown in Fig. 8c).

\section{C) Channel modelling}

The channel sounder provides received power for every TX-RX beam configuration, from which we obtain the double-directional radio channel. Based on the received power and EIRP we obtain measured path loss for distances ranging from 1.5 to $14.25 \mathrm{~m}$. For the LOS measurements, we fit the minimum measured PL value, corresponding to an AoA and AoD of $0^{\circ}$, for each distance to the oneslope model presented in (1). The regression parameters are $P L_{0}$ (in $\mathrm{dB}$ ), the PL at a reference distance $d_{0}$ of $1.5 \mathrm{~m}$ (as in [39]), and PL exponent $n(-)$. The shadow fading term $\chi$ (in $\mathrm{dB}$ ) results from a zero-mean normal distribution.

$$
P L(d)=P L_{0}+10 n \log _{10}\left(d / d_{0}\right)+\chi
$$

In order to model NLOS path loss in the vessel, we determine first order reflected components in the PLAP of both LOS and NLOS measurements. For the LOS measurement tracks, the first order reflections are obtained by selecting the local minimum in the PLAP for which the AoD is close to the additive inverse of the AoA and with a manual verification that the angle corresponds to the room geometry. The additional signal attenuation of this reflected path compared to the LOS path is caused by a longer path distance and by a reflection loss which depends on the incident angle and material properties. We calculate the distance of the reflected path via (2), with $d_{\text {LOS }}$ the distance of the LOS path and $\alpha$ the average of the AoA and inverse AoD.

$$
d_{\text {reflected }}=d_{\mathrm{LOS}} / \cos (\alpha)
$$

For the NLOS measurements, we obtain the distance and incident angle from the room geometry and the locations of the TX and RX nodes. We obtain the reflection loss by subtracting the PL, calculated via (1) using the reflected path distance, from the measured PL. Even though most surfaces in the vessel are metallic, i.e. machinery, floor and ceiling, the reflection loss is non-zero.

\section{D) Link budget}

Based on the developed channel models we can predict throughput of the wireless link using the link budget equation presented in (3). The link budget returns the received power $P_{R}$ (in $\mathrm{dBm}$ ) as a function of transmit power $P_{T}$ (in $\left.\mathrm{dBm}\right)$, transmit and receiving antenna gains $G_{T}$ and $G_{R}$ (in $\mathrm{dBi}$ ) and losses $L_{T}, L_{R}$ (in $\mathrm{dB}$ ), as well as the path loss $P L$ (in $\mathrm{dB}$ ). The received power should be higher than the receiver sensitivity $P_{R S}$ (in $\mathrm{dBm}$ ) in order to obtain a certain data rate. Receiver sensitivity of the radio depends on the modulation and coding scheme (MCS) and therefore defines the maximum throughput that can be obtained for a fixed distance and transmit power. Receiver sensitivities for IEEE 802.11ad using a single carrier PHY are listed in Table 1 for different MCS.

$$
P_{R}=P_{T}+G_{T}+G_{R}-L_{T}-L_{R}-P L
$$


Table 1. Receiver sensitivity $\left(P_{R S}\right)$ and data rate (DR) as a function of modulation and coding scheme (MCS) for an IEEE 802.11ad radio [50]

\begin{tabular}{lccccccccccccc}
\hline MCS & 0 & 1 & 2 & 3 & 4 & 5 & 6 & 7 & 8 & 9 & 10 & 11 & 12 \\
\hline$P_{R S}[\mathbf{d B m}]$ & -78 & -68 & -66 & -64 & -64 & -62 & -63 & -62 & -61 & -59 & -55 & -54 & -53 \\
DR [Mbps] & 27.5 & 385 & 770 & 962.5 & 1155 & 1251 & 1540 & 1925 & 2310 & 2502 & 3080 & 3850 & 4620 \\
\hline
\end{tabular}

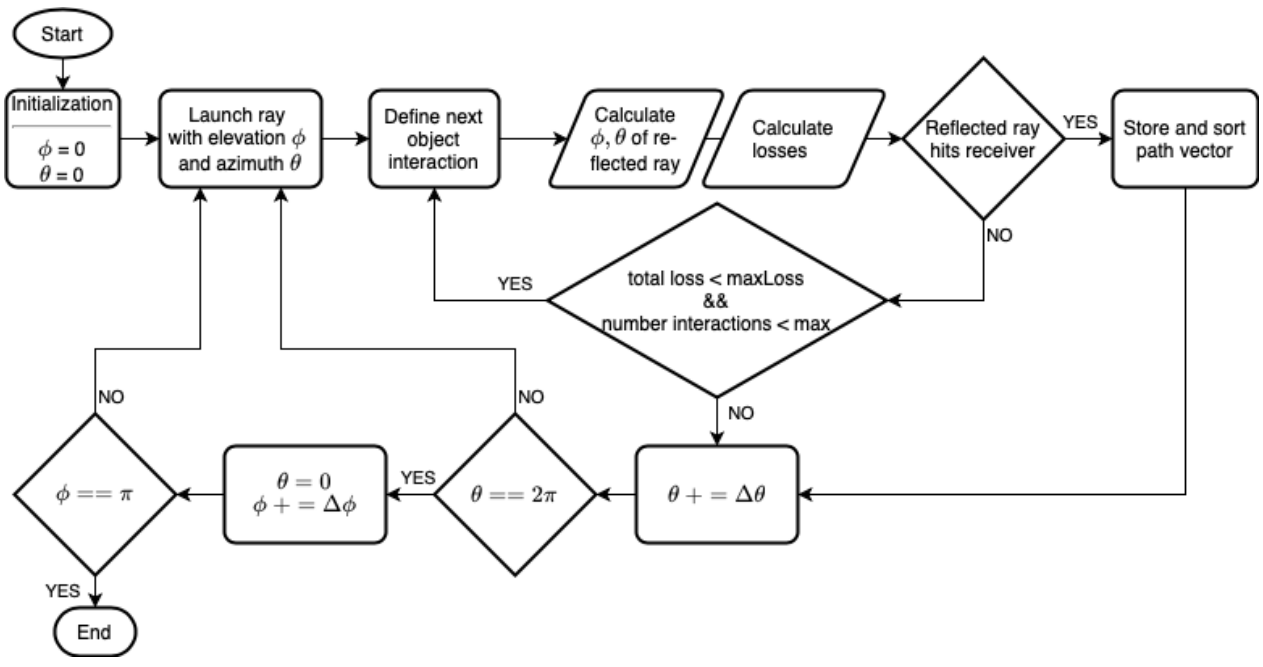

Fig. 3.: Flowchart presentation of ray launching algorithm.

\section{E) Quality of service prediction tool}

The channel model and radio characteristics are implemented in a quality of service $(\mathrm{QoS})$ prediction tool for indoor wireless propagation [51], which calculates received power at every location based on stochastic channel models and material properties. Throughput is calculated via the link budget equation (3). The tool also includes a heuristic network planning algorithm which proposes access point locations that provide full coverage.

\section{1) Algorithm}

We have extended the prediction tool to include a 3-dimensional ray launching algorithm for directive mmWave communication. Via the QoS prediction tool, we estimate coverage based on the predefined location of access points. The environment is first discretized into a finite number of grid points and an access point is placed on the floorplan. At every grid point, a path information vector is stored with a configurable number of best paths to cover the grid point. A path is described by its interactions with the objects in the environment in order to reach the grid point. The path information vector not only stores the path loss associated to the paths, but also their distance, AoA and AoD. For each grid point, rays are launched in the azimuth and elevation plane from the access point, using a spherical coordinate system and with a configurable angle step size.

A flowchart of the ray launching methodology is presented in Fig. 3. The parameters of the algorithm are listed in Table 2. The azimuth angle $\theta$ ranges from 0 to $2 \pi$ whereas elevation angle $\phi$ ranges from 0 to $\pi$. For each ray
Table 2. Ray launching algorithm parameter configuration

\begin{tabular}{lc}
\hline Parameter & Value \\
\hline Elevation increment $\Delta \phi[\mathrm{rad}]$ & $\pi / 100$ \\
Azimuth increment $\Delta \theta[\mathrm{rad}]$ & $\pi / 100$ \\
Grid point size [m] & 0.5 \\
Maximum loss [dB] & 200 \\
Maximum number of reflections & 5 \\
\hline
\end{tabular}

we define the interaction with the environment and calculate the associated losses, including PL and reflection loss. As the objects in the environment block communication, no transmission losses are taken into account. Path and reflection loss are calculated based on the channel model from Sect. II-C. If the reflected ray reaches the accounted grid point, we store the path information vector and sort all path vectors of the grid point according to total loss. A ray is considered to reach a grid point if passes within $0.1 \mathrm{~m}$ from the grid point. If the ray does not reach the considered grid point, we define the next object interaction if the total loss is below the adjustable maximum path loss and if the maximum number of interactions has not yet been reached. We take into account reflection on metallic objects, as well as the floor and ceiling.

We launch rays from every possible angle and do not consider the directivity of the TX antenna. Due to the narrow beam width of actual high-gain mmWave antennas, we define the path loss at every grid point as the path loss associated to the best path and we do not sum the path loss of the different paths as we would typically do for lower frequencies where omnidirectional antennas are used. The 


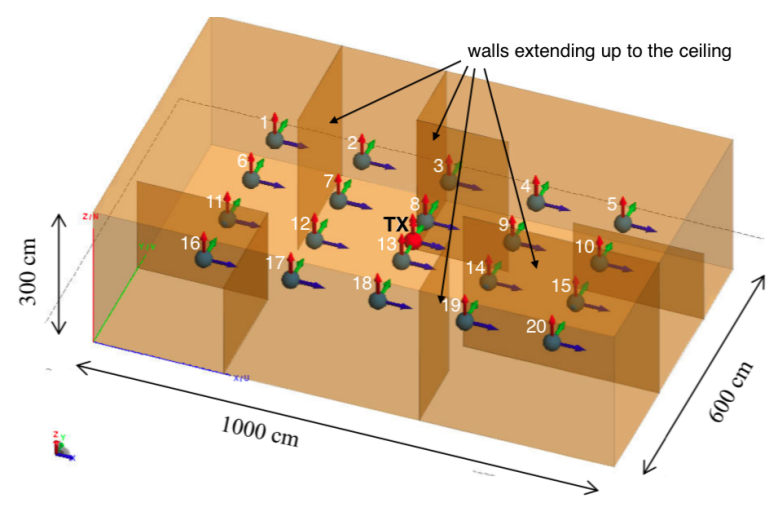

Fig. 4.: Room measuring 10 by 6 m modelled in FEKO, with 1 transmitter at the center of the room and 20 receiver locations at height $1.5 \mathrm{~m}$.

resulting coverage data should be interpreted as the maximum received power if the antennas are steered in the direction of the best path. Even though we associate the received power of the best path for each grid point, we store the best paths in order to compare the power received via the reflected path to the power of the LOS path. By using the best path for the PL calculation we can speed up the simulation time as for grid points with a LOS path to the access point we do not need to use the ray launching algorithm.

\section{2) Validation}

The ray launching algorithm has been validated by comparing simulation results to the commercial analytic electromagnetic wave (EM) solver FEKO based on the Method of Moments integral formulation of Maxwell's equations in combination with the uniform theory of diffraction. We compare the received power at different locations in a metallic room measuring 10 by $6 \mathrm{~m}$, modelled as a perfect electrical conductor. The solver simulation takes into account direct, reflected and diffracted paths. We perform simulations for 15 frequencies within a single IEEE 802.11ad channel and average the received power. We selected single precision data storage and enabled both normal geometry and mesh element size checks. The room with the receiver locations is shown in Fig. 4. An identical room is modelled in our prediction tool. Our prediction tool uses the channel model from Sect. II-C, i.e. the distancerelated path loss is found via the LOS path loss model and the reflection loss uses the NLOS model. In order to validate our ray launching implementation via the FEKO simulation, we use the theoretic path loss according to Friis formula and assume zero reflection losses in the ray launching algorithm. We compare the received power of both simulations at all receiver locations.

The results from FEKO show a high frequency dependency, which is caused by the omnidirectional antennas and the low coherence bandwidth which results in a pronounced multipath fading effect [52]. Figure 5 shows the

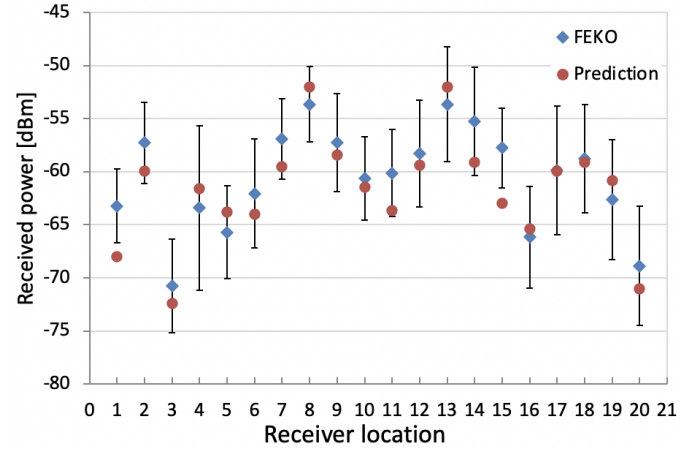

Fig. 5.: Predicted received power and simulated received power via the analytical solver for all receiver locations shown in Fig. 4.

predicted received power for each of the receiver locations from Fig. 4 as well as the simulated received power we obtained from the FEKO simulations, averaged over the full $2.2 \mathrm{GHz}$ band of the IEEE 802.11ad channel and indicating the standard deviation of the 15 simulations.

The predicted received power is within the standard deviation of the FEKO simulations for all receiver locations except location 1 . The average difference between the predicted power and the average of the FEKO simulations is $1.9 \mathrm{~dB}$. The locations for which the predicted received power is significantly below the received power from the simulations seem to correspond to locations where the receiver is in the shadow zone of a (metallic) wall. Our ray launching algorithm does not take into account diffracted rays; as FEKO takes into account diffraction there is a higher received power.

\section{RESULTS}

In this section, we start with presenting the LOS and NLOS channel models. Based on these channel models we provide a link budget analysis in Section III-C. In Section III-D we first provide the validation of the ray launching algorithm after which we present the QoS prediction analysis for the vessel.

\section{A) Line-of-sight channel model}

In order to illustrate a typical PLAP for a LOS measurement in the vessel, Fig. 6 shows the PLAP for 2 distances at measurement track 1 . There is a strong LOS component for both AoA and AoD around $0^{\circ}$, and in Fig. $6 \mathrm{~b}$ two reflected components are present at AoA and AoD around $\pm 10^{\circ}$ and around $\pm 20^{\circ}$. Figure 7 presents the minimum measured PL values as a function of distance for the different LOS measurement tracks shown in Fig. 2, together with the oneslope LOS model of the combined data set (dashed blue line) as well as free space PL (solid line). The circle colors represent the different measurement tracks. Also shown in the figure are the NLOS PL samples that we obtained from the LOS measurements' PLAPs. 


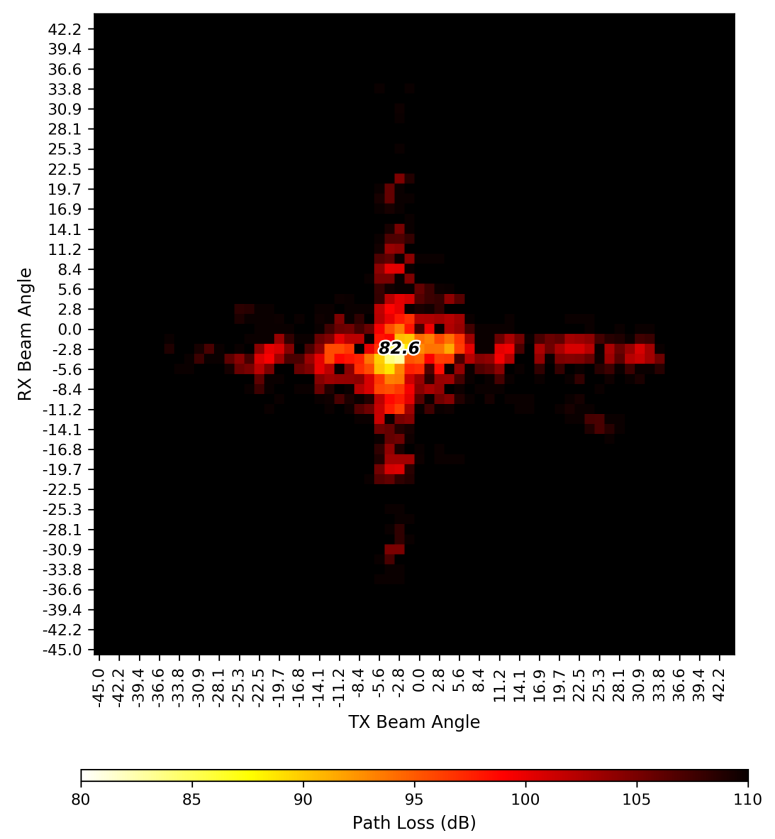

(a) Distance $2.75 \mathrm{~m}$

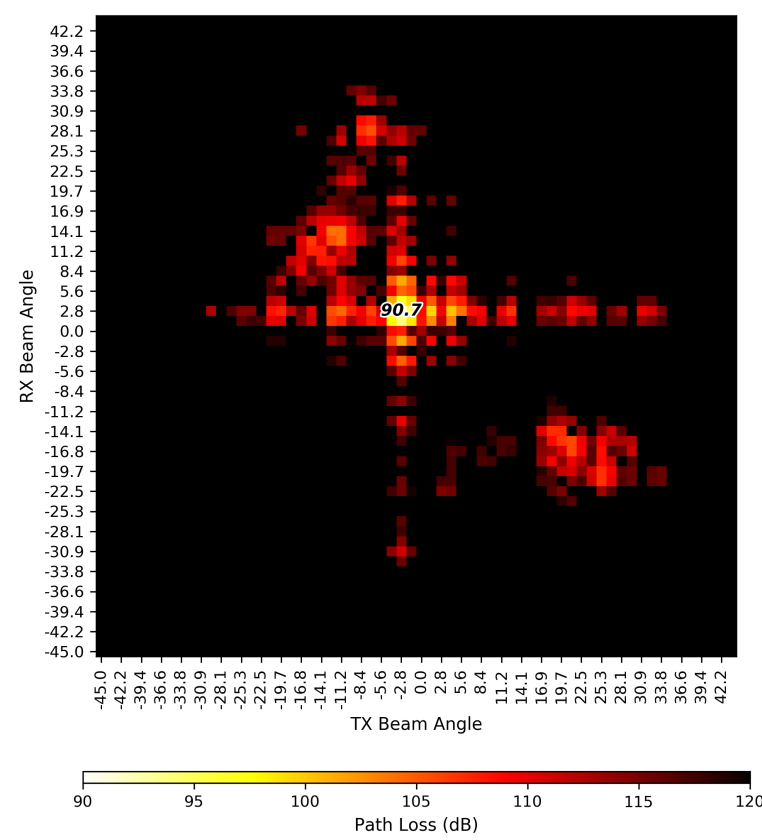

(b) Distance $8.75 \mathrm{~m}$

Fig. 6.: Path loss angular profile (PLAP). Measured path loss as a function of angle of arrival (AoA) and angle of departure $(\mathrm{AoD})$ for Line-of-Sight measurement track 1.

The fitted path loss $P L_{0}$ at reference distance $d_{0}=1.5$ $\mathrm{m}$ is $74.6 \mathrm{~dB}$, which is higher than the free space PL of $71.6 \mathrm{~dB}$ at the same reference distance. However, the fitted PL exponent $n$ of 1.68 is lower than the free space PL exponent of 2. The root mean squared error (RMSE) between the model and measurement data is $3.48 \mathrm{~dB}$, which results in an added $95 \%$ shadow margin of $5.8 \mathrm{~dB}$. The shadow margin added to the PL is shown in Fig. 7 as the red dotted line. For measurement track 1, which is parallel to the wall furthest away from the main engine, the measured PL is around $5 \mathrm{~dB}$ higher than free space PL, whereas the measured PL for locations 2 to 5 is close to or lower than free space PL.

Based on the CIR estimation we obtain the RMS delay for every beam configuration. The average RMS delay for the significant taps of the CIR of the LOS beams is $2.96 \mathrm{~ns}$ and the standard deviation is $3.5 \mathrm{~ns}$.

\section{B) Non line-of-sight channel model}

From the PLAP of the LOS measurements shown in Fig. 6, we conclude that next to the strong LOS component, reflections are received. As the TX and RX angles are similar with opposite signs, and due to the geometry of the room, we suppose that these are first-order reflections. We also notice local minima in the PLAP for beam configurations with equal TX and RX angles, which are assumed to be 2nd order reflections. In order to assess the feasibility of NLOS communication from the LOS measurements, we select the main reflected paths with the lowest measured PL for which the absolute values of the TX and RX angles are approximately the same. The measured PL of the selected reflected path is visualized in Fig. 7 by diamond symbols.

We calculate the distance of these reflected paths via (2); but as the angles of arrival and departure are relatively small, the reflected path distances are close to the LOS path distance and we conclude that the additional path loss of the reflected path is mainly caused by reflection loss, rather than the loss caused by an increased distance. Figure 8 shows the PLAP for two NLOS measurements. For all the NLOS measurements, we determine the distance and incident angle of the reflected paths based on the vessel's floorplan, and calculate reflection loss as a function of incident angle by subtracting the LOS path loss using the reflected path distance from the measured PL. The reflection losses for all measurements as a function of incident angle are shown in Fig. 9, and for each reflection we indicate whether it is more likely to be a first-order or second-order reflection. The added loss of the first-order reflected paths is $16.0 \mathrm{~dB}$, which increases up to $19.7 \mathrm{~dB}$ for the paths that are assumed to correspond to a secondorder reflection. We do not see the angle-dependence that we would expect from the Fresnel reflection coefficient, and the reflection loss is larger than what we expect in a metallic environment. We can therefore assume that multiple close reflections occur due to the irregular shapes of the engine room's objects causing intra-cluster components. From a deployment perspective it is clear that losses up to $30 \mathrm{~dB}$ need to be accounted for when using NLOS paths for communication.

The average RMS delay spread of the NLOS paths slightly increases to $4.32 \mathrm{~ns}$, whereas the standard deviation remains $3.4 \mathrm{~ns}$. The low RMS delay spread was expected as the channel sounder uses a narrow beam width. Figure 10 shows the histogram of all RMS delay spread data for both 


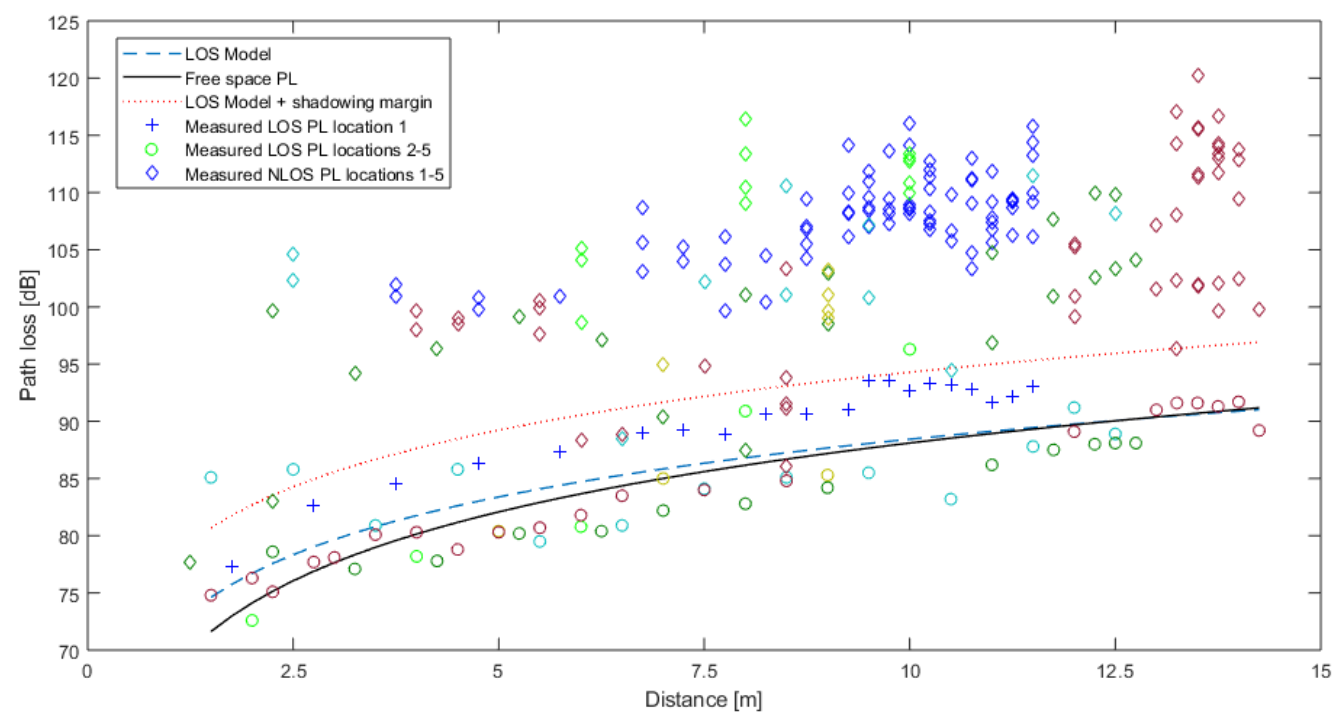

Fig. 7.: Measured Line-of-Sight (LOS) and non Line-of-Sight (NLOS) path loss (PL) from the LOS measurement tracks, with LOS PL model for the engine room of a vessel as well as the free space PL. The different tracks are visualized with different colors; the LOS measurement data is visualized with a circle marker, and the NLOS data with a diamond symbol. The LOS data of track 1 is visualized by the plus symbol.

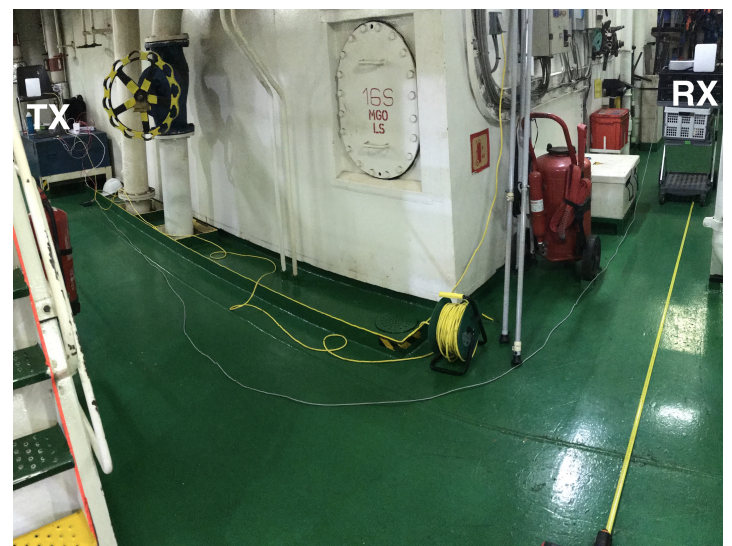

(a) Receiver is behind the corner in engine room

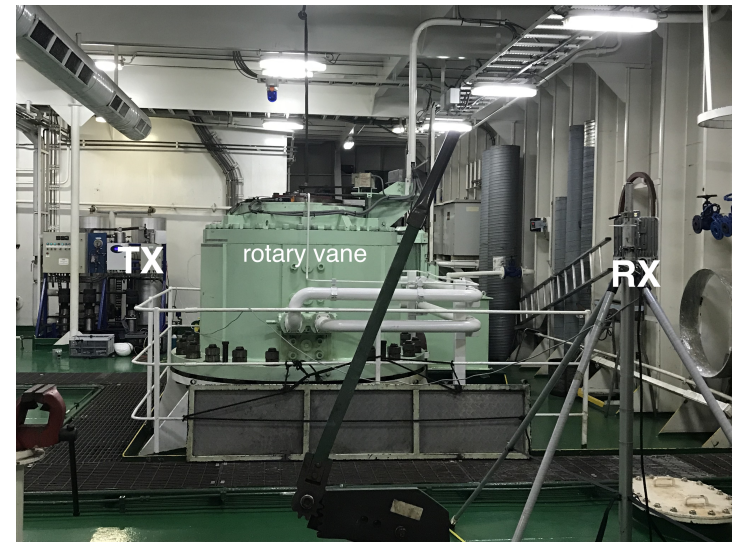

(c) Vane obstructing the Line-of-Sight path in steering gear room

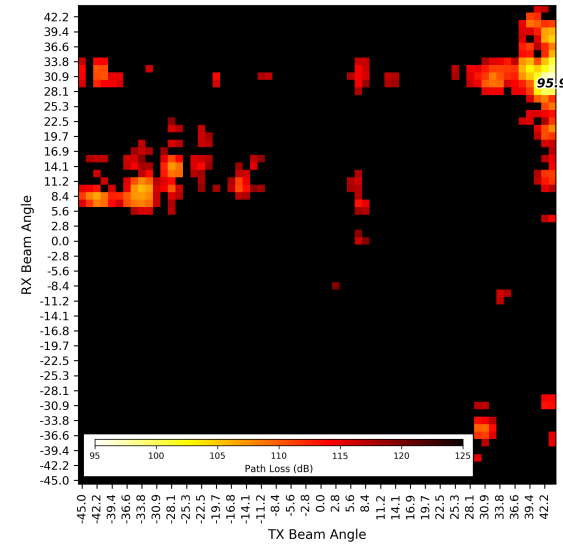

(b) PLAP for corner crossing in engine room

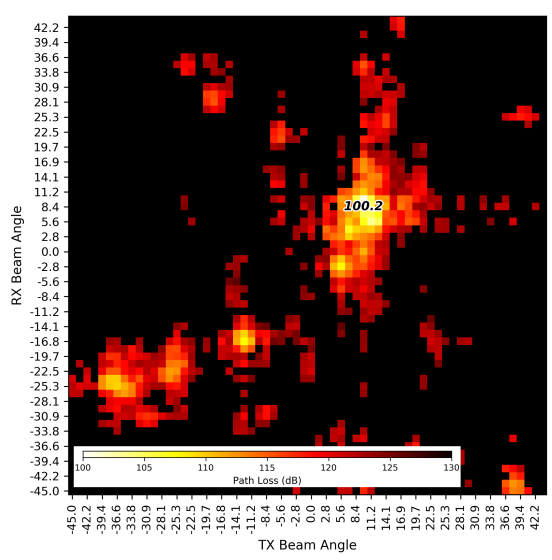

(d) PLAP with wall reflection to bypass vane obstruction

Fig. 8.: Path loss angular profile (PLAP) with measured path loss as a function of angle of arrival (AoA) and angle of departure (AoD) for 2 non Line-of-Sight (NLOS) measurement locations. 


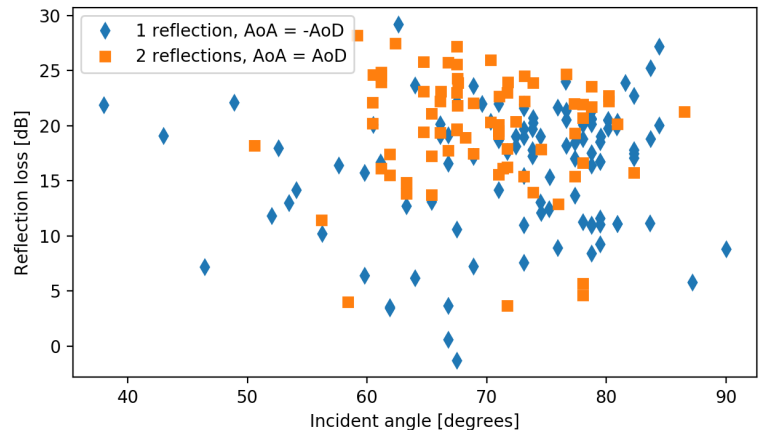

Fig. 9.: Reflection loss as a function of incident angle.

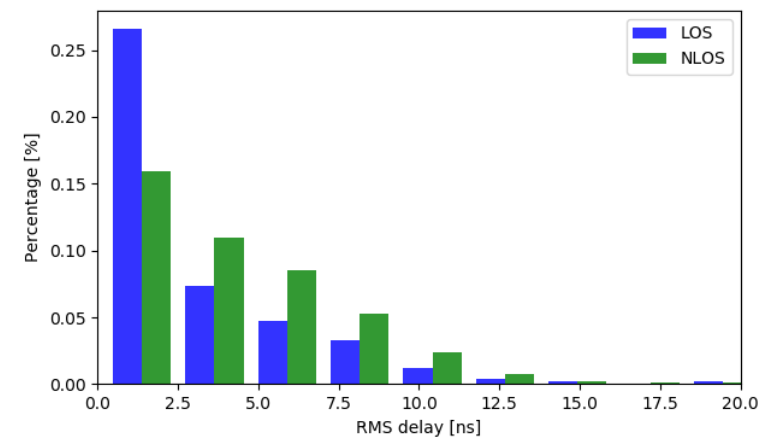

Fig. 10.: Histogram showing percentage of measured RMS delay spread values for both Line-of-Sight and non Lineof-Sight paths.

LOS and NLOS data. The histogram shows that even for NLOS measurement, the main share of paths has an RMS spread below $2.5 \mathrm{~ns}$, even though a higher number of NLOS paths has a higher RMS delay spread compared to the number of LOS paths. On the one hand, this is caused by the specular reflection of a NLOS path, which does not cause a pulse to spread in time. On the other hand, the higher reflection losses that we measured (cf. Fig. 9), and the irregular nature of the engine room's objects cause NLOS paths with intra-cluster multipath, i.e. multiple reflections with reflection points close to each other. However, the path length of these intra-cluster components is similar, resulting in the same delay and therefore not significantly increasing the delay spread.

\section{C) Link budget analysis}

Figure 11 presents throughput (in Mbps) as a function of distance using the link budget equation (3) for both LOS and NLOS scenarios and based on the PL model presented in the first part of this section. For the NLOS scenario, one reflection is considered with an average reflection loss of $16 \mathrm{~dB}$ added to the LOS PL model. We assume a transmit power $P_{T}$ of $13 \mathrm{dBm}$, an RX antenna gain $G_{R}$ of $8 \mathrm{dBi}$, a feeding loss $L_{T}$ of $2.5 \mathrm{~dB}$ and a receiver loss $L_{R}$ of $0 \mathrm{~dB}$. A $5.8 \mathrm{~dB}$ shadow margin is added and the receiver sensitivities that are listed in Table 1 are used. We compare a

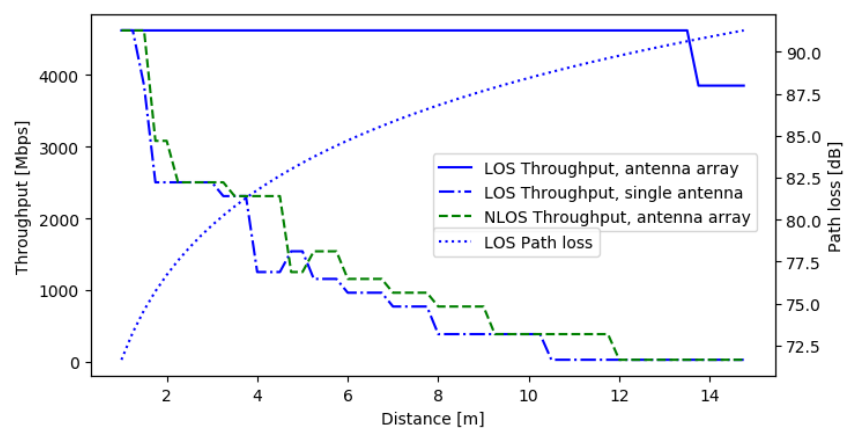

Fig. 11.: Throughput and path loss as a function of distance for a Line-of-Sight and non Line-of-Sight scenario.

single-element patch antenna with a peak gain of $8 \mathrm{dBi}$ as TX antenna to an $8 \times 8$ antenna array with a combined peak gain of $25 \mathrm{dBi}$.

From this link budget, we conclude that using a highgain TX antenna is of utmost importance in order to achieve high data rates. When using a single-element patch antenna with a gain of $8 \mathrm{dBi}$ as TX and RX antenna, the throughput rapidly decreases with distance, and communication via the reflected path is not possible. We see a leap in throughput at distance $5 \mathrm{~m}$ for the LOS scenario with a single patch antenna and at distance $3 \mathrm{~m}$ for the NLOS scenario with the antenna array as TX. This is caused by a constellation change, going from $\frac{\pi}{2}$ quadrature phase shift keying (QPSK) to a $\frac{\pi}{2}$ binary phase shift keying (BPSK), resulting in a higher throughput for the QPSK used at MCS 6 compared to the BPSK from MCS 5, even though the receiver sensitivity using MCS 6 is lower.

\section{D) In-vessel simulations using QoS prediction tool}

We used a free space PL model and assumed perfectly conducting surfaces with zero reflection loss for the validation of the ray launching algorithm. For predicting throughput and coverage via a simulation of received power in the ship hull based on the vessel's floor plan we use the path loss model from Sect. III-A/B. As we use the stochastic channel models, we can model the complex environment by simple geometric objects without the need for millimeter-level accuracy and the materials' conductivity in order to predict received power. The ray launching algorithm is validated for the ship hull environment by comparing specific simulations to measurements from tracks 3 and 4 . We select an access point location identical to the respective transmit location, and inspect the AoD and PL of the simulated best paths for grid points corresponding to receiver node measurement locations. For the validation simulations, the access point and receiver are positioned at the same height of $1.3 \mathrm{~m}$. The LOS component follows the LOS PL model, the simulated AoD of the best reflected path is within 10 degrees of the measured AoD and the PL of the reflected path is within $3 \mathrm{~dB}$ of the measured PL. As the ray launching algorithm launches rays in the 360 degrees azimuth 
and 180 degrees elevation planes, floor and ceiling reflections also appear in the best path, but for tracks 3 and 4 these have a higher associated PL compared to the reflection on the nearby metallic machinery. Figure 12 shows the received power for every grid point at a height of $1.2 \mathrm{~m}$ when one access point is centrally placed at a height of $3 \mathrm{~m}$. The simulation time on a laptop equipped with an Intel Core i5 processor is 325 minutes.

When deploying an actual $60 \mathrm{GHz}$ communication link, only one path will be used for communication from the TX to the RX node as the directive antennas have a narrow beam width. It therefore makes sense to only consider the LOS path when calculating path loss for grid points that have a LOS path to the access point. Furthermore, the simulation time decreases by $65 \%$ when skipping LOS grid points from the ray launching algorithm, compared to applying the ray launching algorithm to all grid points. As most of the objects in the engine room extend almost up to the ceiling, the probability of using a communication link that uses a ceiling reflection is low. We can therefore increase the elevation increment angle $\Delta \phi$ without any significant influence on the prediction outcome. The computation time decreases by $52 \%$ when the elevation step angle is $\pi / 48$ instead of $\pi / 100$. Also increasing the azimuth increment angle $\Delta \theta$ to $\pi / 48$ lowers the computation time by $77 \%$. However, the resolution is lowered and some second-order reflected paths are lost. We use a maximum number of 5 reflections but due to the large reflection loss the received power is lower than the receiver sensitivity when there are more than 2 reflections. According to the link budget example of Sect. $\mathrm{C}$ we could have taken a lower maximum PL of $120 \mathrm{~dB}$ instead of $200 \mathrm{~dB}$, which speeds up the simulation by $60 \%$. It should be noted that the ceiling height of the engine room has no influence on the computation time as simulations with lower ceiling height have similar computation time.

If we calculate path loss of all best paths for the grid points with a LOS component and analyze the share of the power of the best reflected component to the total power of the LOS and reflected components, we see that for $57 \%$ of the grid points, no significant power is received via the reflected path. For the remaining $43 \%$ LOS grid points, the received power from the best reflected path accounts, on average, for $4.5 \%$ of the total received power, with a standard deviation of $1.5 \%$.

\section{CONCLUSION}

The maritime industry is transitioning into a smart transportation ecosystem where interconnected devices are automatically monitored and remotely controlled. Wireless communication is a crucial element of this smart transportation ecosystem. In this paper, we have designed the directive channel model for Line-of-Sight and non Lineof-Sight communication for communication at mmWave frequency $60 \mathrm{GHz}$ in the hull of the ship. Even though the path loss of communication links with a Line-of-Sight

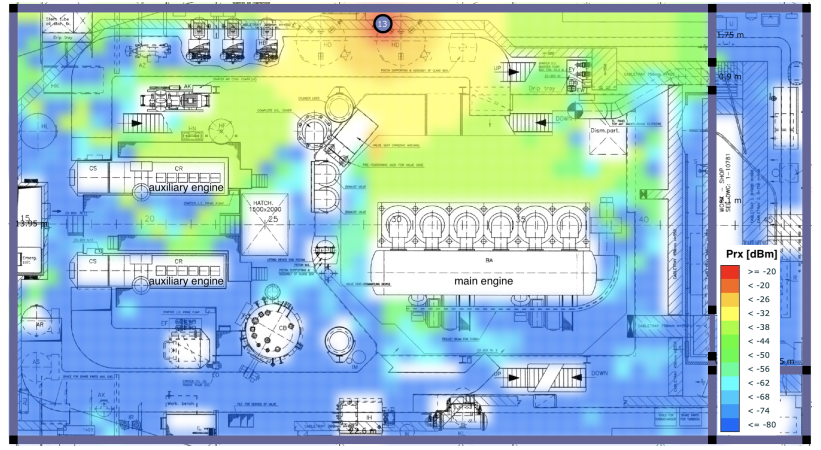

Fig. 12.: Expected received power based on ray launching algorithm and channel model. Using one access point with $13 \mathrm{dBm}$ output power, antenna gain $25 \mathrm{dBi}$ and feeding loss $2.5 \mathrm{~dB}$. The receiver antenna gain is $8 \mathrm{dBi}$. Skipping LOS paths from ray launching algorithm and using settings from Table 2.

path is close to free space path loss, the existence of multiple metallic objects creates severe blockage. On the other hand, there is a considerable reflection loss up to $20 \mathrm{~dB}$, but communication via the first-order reflected path is possible if the total distance is limited. High-gain antennas are necessary and the corresponding narrow beam width results in a low RMS delay spread for both LOS and NLOS links, with an average of respectively 3.0 and $4.3 \mathrm{~ns}$. The channel model is used for throughput and range calculations. A link budget is presented for Line-of-Sight and non Line-of-Sight scenarios. With high-gain antennas we obtain data rates over $3 \mathrm{GHz}$ for Line-of-Sight communication, and data rates over $1 \mathrm{GHz}$ for distances up to $8 \mathrm{~m}$ via a reflected path. We have implemented the channel model in a network prediction tool which can be used to predict received power based on a predefined access point locations for environments similar to the engine and steering gear room of the vessel. For the received power prediction we use a ray launching algorithm which is validated by comparison to an analytic EM solver. The ray launching algorithm takes into account propagation and reflection loss using the stochastic channel models presented in this paper. By using the stochastic channel models, we can predict received power without a detailed 3-D floorplan with millimeter-level machinery dimensions. No transmission through machinery is considered, due to the blocking nature.

For future work, we intend to generalize the ray launching algorithm so it can be used for other environments. Apart from reflection loss and path loss, we will implement partition loss for transmission through an object, as well as diffraction loss around an object. Based on the path information vector, containing object interaction information, we can characterize not only path loss for a certain grid point, but also channel parameters such as delay and angular spread. 


\section{ACKNOWLEDGMENT}

This work was executed within the Internet-of-Shipping (IoS) research project, co-financed by imec with support from Flanders Innovation \& Entrepreneurship. The channel sounder has been granted as part of the Channel Sounder Program of the Telecom Infra Project (TIP). The authors would like to thank Ivan Renette and Franck Ntibashirakandi from Exmar Shipmanagement to facilitate the measurement campaign.

\section{REFERENCES}

[1] W. Honcharenko, H. Bertoni, J. Dailing, J. Qian, and H. Yee, "Mechanisms governing UHF propagation on single floors in modern office buildings," IEEE Transactions on Vehicular Technology, vol. 41, no. 4, pp. 496-504, NOV 1992.

[2] J. Choi, N. Kang, Y. Sung, J. Kang, and S. Kim, "Frequencydependent uwb channel characteristics in office environments," IEEE Transactions on Vehicular Technology, vol. 58, no. 7, pp. 3102-3111, Sep. 2009.

[3] G. R. Maccartney, T. S. Rappaport, S. Sun, and S. Deng, "Indoor office wideband millimeter-wave propagation measurements and channel models at 28 and $73 \mathrm{ghz}$ for ultra-dense $5 \mathrm{~g}$ wireless networks," IEEE Access, vol. 3, pp. 2388-2424, 2015.

[4] X. Wu, C. Wang, J. Sun, J. Huang, R. Feng, Y. Yang, and X. Ge, "60-ghz millimeter-wave channel measurements and modeling for indoor office environments," IEEE Transactions on Antennas and Propagation, vol. 65, no. 4, pp. 1912-1924, April 2017.

[5] Y. L. C. de Jong, J. A. Pugh, M. Bennai, and P. Bouchard, "2.4 to 61 $\mathrm{GHz}$ Multiband Double-Directional Propagation Measurements in Indoor Office Environments," IEEE Transactions on Antennas and Propagation, vol. 66, no. 9, pp. 4806-4820, SEP 2018.

[6] O. Rubio, R. P. Torres, V. M. Rodrigo Penarrocha, J. R. Perez, H. Fernandez, J.-M. Molina-Garcia-Pardo, and J. Reig, "Contribution to the Channel Path Loss and Time-Dispersion Characterization in an Office Environment at $26 \mathrm{GHz}$," ELECTRONICS, vol. 8, no. 11, NOV 2019.

[7] K. I. Pedersen, P. E. Mogensen, and B. H. Fleury, "A stochastic model of the temporal and azimuthal dispersion seen at the base station in outdoor propagation environments," IEEE Transactions on Vehicular Technology, vol. 49, no. 2, pp. 437-447, March 2000.

[8] T. Kurner and A. Meier, "Prediction of outdoor and outdoor-toindoor coverage in urban areas at $1.8 \mathrm{ghz}$," IEEE Journal on Selected Areas in Communications, vol. 20, no. 3, pp. 496-506, April 2002.

[9] Xiongwen Zhao, J. Kivinen, P. Vainikainen, and K. Skog, "Propagation characteristics for wideband outdoor mobile communications at $5.3 \mathrm{ghz}$," IEEE Journal on Selected Areas in Communications, vol. 20, no. 3, pp. 507-514, 2002.

[10] S. Sangodoyin, S. Niranjayan, and A. F. Molisch, "A measurementbased model for outdoor near-ground ultrawideband channels," IEEE Transactions on Antennas and Propagation, vol. 64, no. 2, pp. 740-751, Feb 2016.

[11] V. Kristem, C. U. Bas, R. Wang, and A. F. Molisch, "Outdoor wideband channel measurements and modeling in the 3-18 ghz band," IEEE Transactions on Wireless Communications, vol. 17, no. 7, pp. 4620-4633, 2018
[12] E. Tanghe, W. Joseph, L. Verloock, L. Martens, H. Capoen, K. V. Herwegen, and W. Vantomme, "The industrial indoor channel: largescale and temporal fading at 900, 2400, and $5200 \mathrm{mhz}$,' IEEE Transactions on Wireless Communications, vol. 7, no. 7, pp. 2740-2751, 2008.

[13] E. Tanghe, D. P. Gaillot, M. Liénard, L. Martens, and W. Joseph, "Experimental analysis of dense multipath components in an industrial environment," IEEE Transactions on Antennas and Propagation, vol. 62, no. 7, pp. 3797-3805, 2014.

[14] M. Cheffena, "Propagation Channel Characteristics of Industrial Wireless Sensor Networks," IEEE Antennas and Propagation Magazine, vol. 58, no. 1, pp. 66-73, FEB 2016.

[15] Y. Ai, J. B. Andersen, and M. Cheffena, "Path-loss prediction for an industrial indoor environment based on room electromagnetics," IEEE Transactions on Antennas and Propagation, vol. 65, no. 7, pp. 3664-3674, 2017.

[16] S. Hur, S. Baek, B. Kim, Y. Chang, A. F. Molisch, T. S. Rappaport, K. Haneda, and J. Park, "Proposal on millimeter-wave channel modeling for $5 \mathrm{~g}$ cellular system," IEEE Journal of Selected Topics in Signal Processing, vol. 10, no. 3, pp. 454-469, 2016.

[17] T. S. Rappaport, G. R. MacCartney, S. Sun, H. Yan, and S. Deng, "Small-scale, local area, and transitional millimeter wave propagation for $5 \mathrm{~g}$ communications," IEEE Transactions on Antennas and Propagation, vol. 65, no. 12, pp. 6474-6490, 2017.

[18] T. S. Rappaport, Y. Xing, G. R. MacCartney, A. F. Molisch, E. Mellios, and J. Zhang, "Overview of millimeter wave communications for fifth-generation $(5 \mathrm{~g})$ wireless networks-with a focus on propagation models," IEEE Transactions on Antennas and Propagation, vol. 65 , no. 12 , pp. 6213-6230, 2017.

[19] D. He, B. Ai, K. Guan, J. M. García-Loygorri, L. Tian, Z. Zhong, and A. Hrovat, "Influence of typical railway objects in a mmwave propagation channel," IEEE Transactions on Vehicular Technology, vol. 67, no. 4, pp. 2880-2892, 2018.

[20] I. A. Hemadeh, K. Satyanarayana, M. El-Hajjar, and L. Hanzo, "Millimeter-wave communications: Physical channel models, design considerations, antenna constructions, and link-budget," IEEE Communications Surveys Tutorials, vol. 20, no. 2, pp. 870913,2018

[21] D. Caudill, P. B. Papazian, C. Gentile, J. Chuang, and N. Golmie, "Omnidirectional channel sounder with phased-arrayantennas for $5 \mathrm{~g}$ mobile communications," IEEE Transactions on Microwave Theory and Techniques, vol. 67, no. 7, pp. 2936-2945, 2019.

[22] U. T. Virk and K. Haneda, "Modeling human blockage at $5 \mathrm{~g}$ millimeter-wave frequencies," IEEE Transactions on Antennas and Propagation, vol. 68, no. 3, pp. 2256-2266, March 2020.

[23] M. Z. Aslam, Y. Corre, J. Belschner, G. S. Arockiaraj, and M. Jäger, "Analysis of 60-ghz in-street backhaul channel measurements and lidar ray-based simulations," in 2020 14th European Conference on Antennas and Propagation (EuCAP), 2020, pp. 1-5.

[24] I. Im, D. Shin, and J. Jeong, "Components for smart autonomous ship architecture based on intelligent information technology," Procedia Computer Science, vol. 134, pp. 91-98, 2018, the 15th International Conference on Mobile Systems and Pervasive Computing (MobiSPC 2018) / The 13th International Conference on Future Networks and Communications (FNC-2018) / Affiliated Workshops. [Online]. Available: https://www.sciencedirect.com/ science/article/pii/S1877050918311116

[25] "Electrical installations in ships - part 370: Guidance on the selection of cables for telecommunication and data transfer including radiofrequency cables," International Electrotechnical Commission TR 60092-370, Tech. Rep., 2019. 
[26] J. Parker, "European view of automated vhf/uhf radio systems marine spectrum usage alternatives and trends," IEEE Journal of Oceanic Engineering, vol. 2, no. 3, pp. 239-242, 1977.

[27] S. Ohmori, A. Irimata, H. Morikawa, K. Kondo, Y. Hase, and S. Miura, "Characteristics of sea reflection fading in maritime satellite communications," IEEE Transactions on Antennas and Propagation, vol. 33, no. 8, pp. 838-845, 1985.

[28] M. A. Cervera and A. Ginesi, "On the performance analysis of a satellite-based ais system," in 2008 10th International Workshop on Signal Processing for Space Communications, 2008, pp. 1-8.

[29] F. Bekkadal, "Emerging maritime communications technologies," in 2009 9th International Conference on Intelligent Transport Systems Telecommunications, (ITST), 2009, pp. 358-363.

[30] F. Dong and Y. H. Lee, "Non-line-of-sight communication links over sea surface at 5.5ghz," in Asia-Pacific Microwave Conference 2011, 2011, pp. 1682-1685.

[31] G. Xu, W. Shen, and X. Wang, "Applications of wireless sensor networks in marine environment monitoring: A survey," Sensors, vol. 14, no. 9, pp. 16932-16954, 2014. [Online]. Available: https://www.mdpi.com/1424-8220/14/9/16932

[32] E. Balboni, J. Ford, R. Tingley, K. Toomey, and J. Vytal, "An empirical study of radio propagation aboard naval vessels," in 2000 IEEE-APS Conference on Antennas and Propagation for Wireless Communications (Cat. No.00EX380), 2000, pp. 157-160.

[33] D. R. J. Estes, T. B. Welch, A. A. Sarkady, and H. Whitesel, "Shipboard radio frequency propagation measurements for wireless networks," in 2001 MILCOM Proceedings Communications for Network-Centric Operations: Creating the Information Force (Cat. No.01CH37277), vol. 1, 2001, pp. 247-251 vol.1.

[34] B. De Beelde, E. Tanghe, M. Yusuf, D. Plets, and W. Joseph, "Radio channel modelling in a ship hull: Path loss at $868 \mathrm{mhz}, 2.4 \mathrm{ghz}, 5.25$ ghz and $60 \mathrm{ghz}$,' IEEE Antennas and Wireless Propagation Letters, pp. 1-1, 2021.

[35] H. Kdouh, C. Brousseau, G. Zaharia, G. Grunfeleder, and G. E. Zein, "A realistic experiment of a wireless sensor network on board a vessel," in 2012 9th International Conference on Communications (COMM), 2012, pp. 189-192.

[36] Hao Xu, V. Kukshya, and T. S. Rappaport, "Spatial and temporal characteristics of 60-ghz indoor channels," IEEE Journal on Selected Areas in Communications, vol. 20, no. 3, pp. 620-630, 2002.

[37] N. Moraitis and P. Constantinou, "Indoor channel measurements and characterization at $60 \mathrm{ghz}$ for wireless local area network applications," IEEE Transactions on Antennas and Propagation, vol. 52, no. 12, pp. 3180-3189, 2004.

[38] K. Haneda, J. Järveläinen, A. Karttunen, M. Kyrö, and J. Putkonen, "A statistical spatio-temporal radio channel model for large indoor environments at 60 and $70 \mathrm{ghz}$, , IEEE Transactions on Antennas and Propagation, vol. 63, no. 6, pp. 2694-2704, 2015.

[39] M. Z. Zaaimia, R. Touhami, L. Talbi, M. Nedil, and M. C. E. Yagoub, "60-ghz statistical channel characterization for wireless data centers," IEEE Antennas and Wireless Propagation Letters, vol. 15, pp. 976-979, 2016.

[40] B. De Beelde, E. Tanghe, M. Yusuf, D. Plets, E. De Poorter, and W. Joseph, "60 ghz path loss modelling inside ships," in 2020 14th European Conference on Antennas and Propagation (EuCAP), 2020 , pp. $1-5$

[41] R. Charbonnier, C. Lai, T. Tenoux, D. Caudill, G. Gougeon, J. Senic, C. Gentile, Y. Corre, J. Chuang, and N. Golmie, "Calibration of raytracing with diffuse scattering against 28-ghz directional urban channel measurements," IEEE Transactions on Vehicular Technology, vol. 69 , no. 12 , pp. $14264-14276,2020$
[42] H. Yang, M. H. A. J. Herben, and P. F. M. Smulders, "Indoor radio channel fading analysis via deterministic simulations at $60 \mathrm{ghz}$," in 2006 3rd International Symposium on Wireless Communication Systems, 2006, pp. 144-148.

[43] A. Maltsev, A. Pudeyev, I. Karls, I. Bolotin, G. Morozov, R. Weiler, M. Peter, and W. Keusgen, "Quasi-deterministic approach to mmwave channel modeling in a non-stationary environment," in 2014 IEEE Globecom Workshops (GC Wkshps), 2014, pp. 966-971.

[44] A. Karstensen, Wei Fan, I. Carton, and G. F. Pedersen, "Comparison of ray tracing simulations and channel measurements at mmwave bands for indoor scenarios," in 2016 10th European Conference on Antennas and Propagation (EuCAP), 2016, pp. 1-5.

[45] A. Zhou, J. Huang, J. Sun, Q. Zhu, C. Wang, and Y. Yang, "60 ghz channel measurements and ray tracing modeling in an indoor environment," in 2017 9th International Conference on Wireless Communications and Signal Processing (WCSP), 2017, pp. 1-6.

[46] C. Gentile, P. B. Papazian, R. Sun, J. Senic, and J. Wang, "Quasideterministic channel model parameters for a data center at $60 \mathrm{ghz}$," IEEE Antennas and Wireless Propagation Letters, vol. 17, no. 5, pp. 808-812, 2018

[47] S. Garcia Sanchez, S. Mohanti, D. Jaisinghani, and K. R. Chowdhury, "Millimeter-wave base stations in the sky: An experimental study of uav-to-ground communications," IEEE Transactions on Mobile Computing, pp. 1-1, 2020.

[48] M. H. Tariq, I. Chondroulis, P. Skartsilas, N. Babu, and C. B Papadias, "mmwave massive mimo channel measurements for fixed wireless and smart city applications," in 2020 IEEE 31st Annual International Symposium on Personal, Indoor and Mobile Radio Communications, 2020, pp. 1-6.

[49] S. G. Sanchez and K. R. Chowdhury, "Robust 60ghz beamforming for uavs: Experimental analysis of hovering, blockage and beam selection," IEEE Internet of Things Journal, pp. 1-1, 2020.

[50] IEEE 802.11ad - IEEE Standard for Information technologyTelecommunications and information exchange between systemsLocal and metropolitan area networks-Specific requirements-Part 11: Wireless LAN Medium Access Control (MAC) and Physical Layer (PHY) Specifications Amendment 3: Enhancements for Very High Throughput in the $60 \mathrm{GHz}$ Band. IEEE Computer Society, 2012.

[51] D. Plets, W. Joseph, K. Vanhecke, E. Tanghe, and L. Martens, "Coverage prediction and optimization algorithms for indoor environments," EURASIP Journal on Wireless Communications and Networking, pp. 123:1-123:23, 2012. [Online]. Available: http://dx.doi.org/10.1186/1687-1499-2012-123

[52] Haibing Yang, P. F. M. Smulders, and M. H. A. J. Herben, "Frequency selectivity of 60-ghz los and nlos indoor radio channels," in 2006 IEEE 63rd Vehicular Technology Conference, vol. 6, 2006, pp. 2727-2731

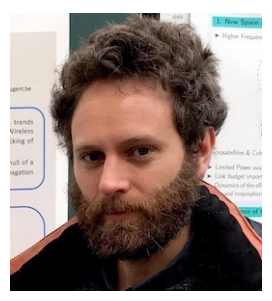

Brecht De Beelde received a M.Sc degree in Electrical Engineering from Ghent University (Belgium) in 2013. He started his career as an embedded SW engineer at the Low-Power Wireless Division of Qorvo, Inc where he worked on BLE and ZigBee communication controllers. He joined the WAVES research group of the Department of Information Technology (INTEC) of Ghent University in 2018, where he is currently working towards a $\mathrm{PhD}$ degree. His research interests are channel modelling and wireless network planning in industrial environments and at mmWave and sub-Terahertz frequencies. 


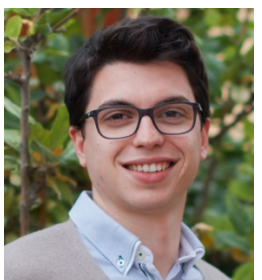

Andrés Almarcha Lopéz received his degree in Telecommunication Engineering from the Technical University of Cartagena (Spain) in 2015. $\mathrm{He}$ started his career as validation engineer at NXP Smart Antennas Solutions. Prior to joining Barcelona Supercomputing Center in 2021 as a digital verification engineer, he was responsible for the verification of Bluetooth LE SoCs at Dia$\log$ Semiconductor. He currently works on the MareNostrum Exascale Experimental Platform.

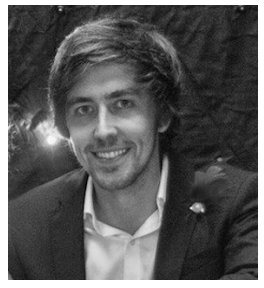

David Plets received a M.Sc degree in Electrical Engineering from Ghent University (Belgium) in 2006 and joined the WAVES research group that same year. He obtained his $\mathrm{PhD}$ degree in 2011 and has been Assistant Professor since 2016. His current research interests include localization techniques and IoT for both industry- and health-related applications. He is also involved in the optimization of wireless communication and exposure, and interference. broadcast networks, with a focus on coverage,

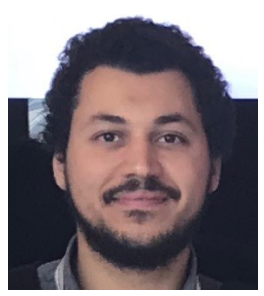

Marwan Yusuf received the M. Sc. degree in Electrical Engineering from Istanbul Medipol University (Turkey) in 2016 where he was a Research Assistant in the Communications, Signal Processing, and Networking Center (CoSiNC). $\mathrm{He}$ joined the WAVES research group 2018 and is currently working towards a $\mathrm{PhD}$ degree. His research interests are measurementbased modelling of indoor and outdoor wireless propagation with emphasis on vehicular commu-

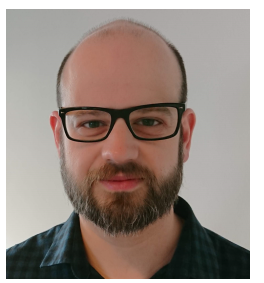

Emmeric Tanghe received the M. Sc. degree in Electrical Engineering from Ghent University (Belgium) in July 2005 and joined the WAVES research group that same year. He obtained his $\mathrm{PhD}$ degree in 2011 with a dissertation on the modelling of indoor and outdoor propagation through field measurements. He became a parttime professor in medical applications of electromagnetic fields in and around the human body in 2015. He continues his work on propagation modelling, covering indoor and outdoor propagation as well as propagation for wireless body area networks and medical implants.

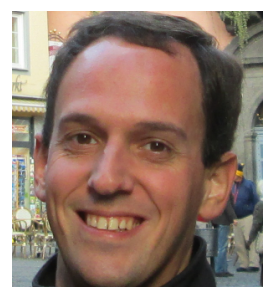

Wout Joseph received the M. Sc. degree in Electrical Engineering from Ghent University (Belgium) in 2000 and joined the WAVES research group that same year. He obtained his $\mathrm{PhD}$ degree in 2005 with a dissertation on measuring and modelling of electromagnetic fields around base stations in order to assess the health effects of the exposure to electromagnetic radiation. Since October 2009, he is professor in the domain of Experimental Characterization of wireless communication systems. His professional interests are electromagnetic field exposure assessment, in-body electromagnetic field modelling, electromagnetic medical applications, propagation for wireless communication systems, IoT, antennas and calibration. Furthermore, he specializes in wireless performance analysis and Quality of Experience.

nication channels. 